\title{
e-Phaïstos
}

e-Phaïstos

Revue d'histoire des techniques / Journal of the history of technology

VI-1 2017 | 2018

Varia

\section{Blanche Segrestin (dir.), Le tournant fayolien}

Jean-Philippe Passaqui

\section{OpenEdition}

Journals

Édition électronique

URL : http://journals.openedition.org/ephaistos/2622

DOI : 10.4000/ephaistos.2622

ISSN : 2552-0741

Éditeur

IHMC - Institut d'histoire moderne et contemporaine (UMR 8066)

Référence électronique

Jean-Philippe Passaqui, «Blanche Segrestin (dir.), Le tournant fayolien», e-Phaïstos [En ligne], VI-1

2017 | 2018, mis en ligne le 27 juin 2018, consulté le 09 novembre 2019. URL : http://

journals.openedition.org/ephaistos/2622

Ce document a été généré automatiquement le 9 novembre 2019.

Tous droits réservés 


\title{
Blanche Segrestin (dir.), Le tournant fayolien
}

\author{
Jean-Philippe Passaqui
}

\section{RÉFÉRENCE}

SEGRESTIN Blanche (dir.), « Le tournant fayolien », Entreprises et histoire, juin 2016, n83, p. 5 à 136.

1 L'année 2016 a été marquée par plusieurs initiatives s'inscrivant dans le cadre de la commémoration du $100^{\mathrm{e}}$ anniversaire de la publication d'Administration Industrielle et Générale (AIG), par Henri Fayol, sous la forme d'un article inséré dans le Bulletin de la Société de l'industrie minérale, société d'ingénieurs, de techniciens de la mine et des industries métallurgiques, dont le siège se trouvait à Saint-Étienne ${ }^{1}$. Fayol, ingénieur civil des mines de formation, issu de l'École des mines de Saint-Étienne (EMSE), directeur d'une des plus grandes entreprises minières et métallurgiques de France, en était un des membres les plus éminents. Il est d'abord connu pour ses publications, tardives au regard de sa carrière, en gestion et management de l'entreprise.

2 Parmi les différentes actions et manifestations, un colloque international, s'inscrivant aussi dans le cadre du Bicentenaire de la création de l'École des mines de Saint-Étienne, a réuni des historiens, des sociologues, des gestionnaires, mais aussi des ingénieurs des mines et, plus généralement, des spécialistes des sciences de la terre. Intitulé « Henri Fayol, histoire, économie, sciences ", il s'est tenu à Saint-Étienne les $1^{\mathrm{er}}, 2$ et 3 juin 2016 ${ }^{2}$. Au même moment, avec d'ailleurs des partenaires conjoints, la revue Entreprises et histoire consacrait son numéro de juin 2016 au « Tournant fayolien », pour reprendre le terme utilisé par Blanche Segrestin dans l'éditorial de cet ensemble d'articles proposés par des spécialistes de la pensée fayolienne ${ }^{3}$. Pourquoi ce regain d'intérêt qui dépasse et de loin le simple aspect commémoratif?

3 Dans un contexte marqué par la remise en cause de la place du manager dans l'entreprise, face à des actionnaires au poids grandissant, Fayol apparaît à nouveau 
comme un personnage actuel, moderne. Certains aspects du capitalisme actuel présentent des caractéristiques communes à la situation contemporaine de Fayol, ainsi que le rappelle A. Hatchuel: "Le capitalisme financier a provoqué une étrange régression anti-fayolienne dans un monde qui n'avait jamais été aussi fayolien. La dimension créative n'a jamais été aussi nécessaire et présente dans la compétition contemporaine par l'innovation. De même que la dimension politique de l'action du chef d'entreprise s'étend à un "corps social" qui va au-delà des personnels et des actionnaires, et inclut des partenaires, des territoires, des États, et des milieux écologiques. Le concept de perfectionnement est passé de l'histoire humaine à celle de la planète ${ }^{4}$.» Le propos de Fayol conserverait donc toute sa modernité. Outre l'éditorial, plusieurs articles retiennent plus particulièrement l'attention, car ils exposent les travaux en cours de plusieurs membres ou collaborateurs ponctuels de l'Équipe d'histoire des techniques.

4 L'association entre Marco Bertilorenzi et Anne-Françoise Garçon semblait incontournable. D'elle est né, en 2014, avec l'appui de leurs recherches antérieures, le colloque intitulé «Entre technique et gestion, une histoire des ingénieurs civils des mines, $\mathrm{XIX}^{\mathrm{e}-\mathrm{XX}}$ siècles ». Dans leur article « De la pratique à une science ? La "nouvelle doctrine" d'Henri Fayol dans son contexte conceptuel ${ }^{5}$ ", Marco Bertilorenzi et AnneFrançoise Garçon insistent sur le fait qu'au cours des années qui ont précédé la publication d'AIG, d'autres grands patrons français ont eux aussi tâché de comprendre l'entreprise et les moyens d'assurer sa bonne gestion, tout en réfléchissant à son développement. Ces hommes partagent une formation commune, sur laquelle nous nous sommes d'ailleurs déjà appesantis. Qu'ils soient sortis des rangs de Paris ou de Saint-Étienne, c'est dans le cadre des Écoles des mines qu'ils ont acquis les compétences nécessaires à affronter l'entreprise pour la diriger, à un moment où celle-ci était sortie de sa phase de création, de développement, pour plutôt se consacrer au maintien des positions industrielles et commerciales établies. À ce propos, la comparaison entre Jean-Baptiste Marsaut, à la tête des houillères de Bessèges, et Henri Fayol, au sein de Commentry-Fourchambault, est riche de sens. Issus tous les deux de l'École des mines de Saint-Étienne, ils ont effectué toute leur carrière au sein de l'entreprise qui les avait recrutés à la sortie de l'école. Cette fidélité est loin d'être systématique. Tous les deux se sont aussi placés, pendant plusieurs décennies, dans les pas de grands capitaines de l'industrie, Chalmeton pour Marsaut, et Mony pour Fayol. À Bessèges comme à Commentry, les caractéristiques productives particulières qui se manifestent dans l'exploitation de la houille ont conduit les industriels, les ingénieurs des mines, civils comme d'État, à se confronter à un environnement complexe, dangereux, où la part de l'incertitude doit être réduite à néant, sous peine de mettre en danger les ouvriers, mais aussi les installations minières et in fine la production. D'ailleurs, signe de la nécessité de cet ordre productif hiérarchisé et discipliné, au moment de quitter la tête des exploitations minières de Commentry-Fourchambault pour devenir directeur général de la même entreprise, Henri Fayol laisse à ses successeurs un document remarquable qui n'est pas sans poser les premiers jalons d'AIG, à savoir son: "Organisation du service technique des houillères de Commentry et de Montvicq", avec un principe très fort: "(assigner) à chaque agent ses fonctions, ses tâches spécifiques et sa position hiérarchique ${ }^{6}{ }^{\prime}$. Il n'y a pourtant pas d'urgence puisque ceux qui assurent la relève étaient, quelques mois plus tôt, ses lieutenants. Fayol les avait choisis avec soin, patiemment formé. Souvent Stéphanois, ils avaient, avant même d'être recrutés au sein de Commentry-Fourchambault, été façonnés par les principes et 
préceptes inculqués par l'EMSE. Le frère d'Henri Fayol, Paul, qui n'était pas sans talents, faisait d'ailleurs partie du lot.

5 Les écrits de François Duffaut sont aussi familiers des lecteurs d'e-Phaïstos. Après avoir participé en 2009 à une séance du séminaire « Énergie et matériaux » consacré à l'acier, il poursuit cette veine " découverte » il y a quelques années, notamment dans le cadre des travaux qu'il conduit au sein du Centre d'études de la métallurgie nivernaise ${ }^{7}$. Ingénieur civil des mines issu de l'École des mines de Paris, ancien directeur R\&D d'Imphy SA et, de ce fait, lointain successeur de Chevenard à la tête du laboratoire d'Imphy, il est aussi un collaborateur régulier de revues et de colloques d'historiens. Fin connaisseur de Chevenard ${ }^{8}$, de ses écrits, il insiste sur les conditions de recrutement de celui-ci par Fayol, en 1910, alors que le site d'Imphy bénéficie depuis quelques années de la collaboration avec le savant suisse Charles-Édouard Guillaume, qui devait déboucher sur la découverte, à partir des années 1894-1896, d'une célèbre série d'aciers alliés au nickel et au chrome ${ }^{9}$. Un important programme pluri-annuel d'investissements en faveur de l'usine d'Imphy, engagé à partir de 1897, en découla. François Duffaut rappelle que ces orientations scientifiques et techniques de l'entreprise devaient assurer sa survie et faire, encore aujourd'hui, des usines d'Imphy (Aperam), une référence mondiale dans la production des aciers de précision. L'impulsion donnée par Fayol aux premières recherches de Chevenard est décisive. De discours en articles, Chevenard l'a constamment rappelé au cours de sa carrière de chercheur au sein de l'entreprise, mais aussi comme enseignant au sein de l'EMSE. Chevenard a patiemment étudié les caractéristiques des aciers à haute teneur en nickel. Par la mise au point de nouveaux appareils de mesure, par une formation minutieuse, imaginée de sorte à utiliser au mieux les compétences de chacun, quelle que soit sa place au sein de son laboratoire, il est parvenu à découvrir de nouveaux aciers. François Duffaut rappelle donc bien que, comme l'avaient déjà démontré Werth et Osmond, l'innovation dans la sidérurgie ne doit rien au hasard. Elle découle d'une démarche associant rigueur, patience, répétition des expériences, qualité des données enregistrées et mise au point d'outils ad hoc. Enfin, et c'est un point plus original des travaux de François Duffaut, la démonstration de l'importance de la relation avec la clientèle est démontrée. Il faut savoir l'écouter, la conseiller et l'orienter, pour sentir, par les bruits qui remontent des consommateurs, quels seront les besoins de demain.

6 Avec Jean-Louis Peaucelle, la revue Entreprises et histoire retrouve un de ses collaborateurs réguliers, qui a déjà publié sur Fayol plusieurs articles et ouvrages de référence. Il a d'ailleurs dirigé un numéro consacré à Henri Fayol, en 2003, pour la revue Entreprises et histoire ${ }^{10}$. Cette fois, Jean-Louis Peaucelle s'intéresse à la gestion des grèves par ce dernier ${ }^{11}$. La question est d'importance. En 1892, il reprend un ensemble industriel, celui de Decazeville qui, quelques années plus tôt, s'était signalé par la violence des manifestations ouvrières. Dans un contexte qui s'y prêtait et alors que luimême dut faire des choix face à certains sites historiques dont il ne parvenait pas à maintenir la rentabilité - on pense à la fermeture de la grande usine de Fourchambault en 1901 - Fayol ne peut échapper aux troubles sociaux du temps. Il les a affrontés en faisant ressortir, à nouveau, ses qualités de meneurs d'hommes, mais aussi certains aspects qui se retrouvent dans AIG comme, notamment, sa capacité à disposer d'une information précise avant de décider. Entre 1888 et 1918, par rapport aux autres houillères et grandes usines du Centre de la France, Fayol et son entreprise ont été relativement épargnés par les grèves. L'année 1902 se détache, marquée par des mouvements sociaux qui agitent plusieurs sites, essentiellement les mines, dans un 
contexte de pression à la baisse sur les prix des houilles, dont les effets se font aussi ressentir sur le niveau des salaires. Mais cela correspond à une vague d'ensemble au sein de laquelle des sociétés minières comme Blanzy ou Épinac apparaissent beaucoup plus fortement et durablement touchées. En outre, les grèves frappent, comme ailleurs, les postes et ateliers les plus pénibles, comme les piqueurs au fond et le laminage dans les usines. Il serait sans doute intéressant de comprendre pourquoi l'entreprise Commentry-Fourchambault et Decazeville est parvenue à échapper aux grandes perturbations de l'année 1906. Sous la forme de grèves dures, mais aussi à cause des pratiques de recrutement de la compagnie de Courrières, soucieuse de combler les vides créés par la catastrophe de 1906, les exploitations du Centre et du Midi de la France ont subi une juxtaposition de revendications et de démissions.

7 Moins touché que ses confrères par les grèves, notamment dans les mines, Fayol réagissait de la même manière, lorsqu'il y était confronté, en n'hésitant pas à renvoyer massivement les ouvriers les plus impliqués, voire en fermant un site, comme à Bouxhors, exploitation minière dépendant de Brassac, qui avait d'ailleurs déjà connu pareil sort quelques années plus tôt, quand le site était encore exploité par les Schneider. Plus que la situation sociale, ce sont des opportunités qui s'offrent à Fayol face à des sites dont la rentabilité fait défaut. Mais il n'hésitait pas non plus, par courrier et campagnes d'affiches, à maintenir le contact avec ses ouvriers. Cette expérience a durablement marqué Fayol ; elle explique sans doute un certain parti pris vis-à-vis des monopoles publics dont le fonctionnement a pu lui sembler haché par la présence syndicale et la récurrence des grèves.

8 Lee D. Parker, dans son article «The global Fayol: contemporary management and accounting traces", montre combien la lecture initiale de Fayol a souffert d'une analyse partielle voire partiale, négligeant la richesse de son propos. Ce texte est donc à mettre en relation avec celui liminaire de Blanche Segrestin. En outre, le contexte a desservi Fayol, dans une sphère industrielle où l'influence du Corps des mines est considérable et, avec lui, celle d'Henry Le Chatelier, dont on connaît le rôle décisif dans la diffusion du taylorisme en France. Pour autant, son influence mérite d'être appréciée à l'aune de l'ambition qui fut la sienne, posant les jalons, tout en les associant, de nombreuses disciplines vitales aux entreprises de son temps, mais aussi à celles qui, aujourd'hui, inscrivent leur action productive et commerciale dans des périmètres fortement dilatés par rapport à ceux contemporains de Fayol, sans que pour autant, la pensée de celui-ci ait pu en souffrir, bien au contraire.

9 Dans la continuité de l'article de François Duffaut, Pascal Le Masson et Benoît Weil reviennent sur la collaboration féconde entre Fayol, Guillaume et Chevenard ${ }^{12}$, à l'origine, avant la Première Guerre mondiale, de l'amorce de l'orientation de la vieille entreprise minière et métallurgique que fut Commentry-Fourchambault et Decazeville vers la métallurgie de précision ${ }^{13}$. Les archives Chevenard sont abondantes. Conservées aux archives départementales de la Nièvre, leur consultation aurait été appréciée ${ }^{14}$. Elles contiennent notamment, et bien au-delà des nombreux articles publiés par Chevenard dans les revues des sociétés scientifiques et techniques, les notes et les tapuscrits de nombreux discours qui, s'ils ont tendance à se répéter, offrent un regard très riche sur la doctrine scientifique portée par ce dernier, sur la place de l'ingénieur dans l'entreprise, mais aussi dans la société, thème reposant selon lui, sur l'originalité de l'ingénieur français, issue d'une excellence qui lui permet d'affirmer, avec le sourire, mais aussi le sentiment d'approcher une vérité, que l'ingénieur des mines français est 
le meilleur du monde. Et sur ce point, Chevenard rappelle souvent combien le legs de Fayol fut, pour lui et pour d'autres, décisif.

10 Cet ensemble d'articles est prolongé, de la p. 121 à la p. 131, par un débat articulé autour de la question suivante : l'innovation a-t-elle joué un rôle dans la naissance de l'administrative science?, débat auquel ont participé Gabriel Galvez-Behar, Armand Hatchuel, Odile Henry et Elle S. O'Connor. Enfin, Blanche Segrestin a édité, en l'annotant et le commentant, le document suivant: «L'entreprise, conséquence de la science moderne (1919) ${ }^{15} »$. Ce numéro de la revue Entreprises et histoire est donc, pour ce qui concerne le dossier consacré à Fayol, d'une grande richesse et démontre combien la pensée de ce grand ingénieur civil des mines mérite d'être abordée avec une curiosité renouvelée et, à ce titre, les réflexions menées par Blanche Segrestin et Armand Hatchuel, par Marco Bertilorenzi et Anne-Françoise Garçon, celles poursuivies par François Duffaut, méritent d'être signalées à la fois pour leur relecture de Fayol, mais aussi pour les pistes qu'elles ouvrent ou donnent envie d'ouvrir quant à l'articulation entre histoire des entreprises et histoire des techniques.

\section{NOTES}

1. FAYOL Henri, "Administration industrielle et générale ", Bulletin de la Société de l'industrie minérale, $5^{\mathrm{e}}$ série, vol. 10, $\mathrm{n}^{\circ} 3,1916$, p. 5-162.

2. Les actes de ce colloque sont en cours de préparation et devraient être publiés dans le courant du premier semestre 2018. Ce colloque fait suite à une initiative précédente, menée les 2 et 3 décembre 2014, avec un colloque intitulé «Entre technique et gestion, les ingénieurs civils des mines dans l'industrialisation de la France et d'ailleurs, $\mathrm{XIX}^{\mathrm{e}}-\mathrm{XX}^{\mathrm{e}}$ siècles », organisé à l'Université Paris I Panthéon-Sorbonne, par Marco Bertilorenzi et Jean-Philippe Passaqui. Les actes de ce colloque ont été publiés sous la forme suivante : BERTILORENZI Marco, PASSAQUI Jean-Philippe et GARÇON Anne-Françoise (dir.), Entre technique et gestion, "une histoire des ingénieurs civils des mines ", XIX ${ }^{e}-X X^{e}$ siècles, Paris, Presses des Mines, 2016, 395 p.

3. Ce remarquable texte introductif de SEGRESTIN Blanche, «Le tournant fayolien: des révolutions industrielles à la naissance de l'entreprise moderne ", Entreprises et histoire, $n^{\circ} 83$, p. 5 à 12 , est à compléter avec l'article d'Armand Hatchuel dans le même volume: HATCHUEL Armand, «Henri Fayol et la théorie du chef d'entreprise : une nouvelle figure de l'autorité au tournant du XX siècle », Entreprises et histoire, n'83, juin 2016, p. 108 à 120 et, du même auteur, HATCHUEL Armand \& SEGRESTIN Blanche, "Henri Fayol, théoricien de l'entreprise innovante », in BERTILORENZI Marco, PASSAQUI Jean-Philippe et GARÇON Anne-Françoise (dir.), Entre technique et gestion,... op. cit., p. 315 à 324 .

4. HATCHUEL Armand, « Henri Fayol... art. cit », p. 120.

5. BERTILORENZI Marco \& GARÇON Anne-Françoise, « De la pratique à une science ?... art. cit. », p. 13 à 35.

6. BERTILORENZI Marco \& GARÇON Anne-Françoise, " De la pratique à une science ?... art. cit. », p. 13 à 35.

7. DUFFAUT François, "Pierre Chevenard ou la recherche au cœur de l'entreprise moderne ", Entreprises et histoire, $\mathrm{n}^{\circ} 83$, juin, 2016, p. 64 à 78. 
8. Cf. notamment, DUFFAUT François, «Actualité du concept de métallurgie de précision. Illustration par l'exemple de l'Invar pour «shadow-mask», Actes du colloque Innovations métallurgiques en Nivernais, 1996, p. 21 à 42. DUFFAUT François, «La métallurgie de précision de Pierre Chevenard ", Le Marteau pilon, t. XXIV, 2012, p. 35 à 44.

9. Par exemple, l'acier NC4 possède une teneur de $24,96 \%$ de nickel et 2,88\% de chrome. Réalisé à la demande de l'Artillerie, il s'est avéré particulièrement adapté à l'obtention de plaques de blindage pour la Marine. ADENOT Hubert (avec l'aide de François Duffaut), «Jacques Adenot, ingénieur civil des mines, 1862-1924 », Le Marteau pilon, t. XXIX, juillet 2017.

10. PEAUCELLE Jean-Louis (dir.), « Henri Fayol », Entreprises et histoire, n³4, décembre 2003 et, plus récemment, PEAUCELLE Jean-Louis et GUTHRIE Cameron, Henri Fayol, the manager, Londres, Pickering et Chatto, 2015.

11. PEAUCELLE Jean-Louis, « Henri Fayol face aux grèves ", Entreprises et histoire, nº3, juin, 2016, p. 36 à 50 .

12. Le MASSON Pascal \& WEIL Benoît, «Fayol, Guillaume et Chevenard, la science, l'industrie et l'exploration de l'inconnu: logique et gouvernance d'une recherche cognitive », Entreprises et histoire, $\mathrm{n}^{\circ}$ 83, juin 2016, p. 79 à 107.

13. Voir à ce sujet, PASSAQUI Jean-Philippe, «De l'acier aux aciers spéciaux, des aciers spéciaux aux aciers haute teneur en nickel », Le Marteau pilon, t. XXIV, 2012, p. 23 à 34.

14. Le corpus documentaire utilisé pour cet article provient de textes de Chevenard qui figurent sur le site internet annales.org et qui reposent sur l'article suivant: PASSAQUI Jean-Philippe et DUFFAUT François, "Textes présentant la démarche scientifique de Pierre Chevenard", Le marteau pilon, t. XXII, 2010, p. 67 à 88.

15. Document extrait de l'ouvrage suivant, préfacé par H. Fayol : WILBOIS Joseph \& VANUXEM Paul, Essai sur la conduite des affaires et la direction des hommes, Paris, Payot, 1919.

\section{INDEX}

Thèmes : Un ouvrage nous a appris

\section{AUTEURS}

\section{JEAN-PHILIPPE PASSAQUI}

Jean-Philippe PASSAQUI est chercheur affilié au Centre d'histoire des techniques, IHMC UMR 8066, Paris I Panthéon-Sorbonne - ENS Ulm - CNRS. Agrégé d'histoire et docteur en histoire contemporaine, il a obtenu le prix spécial d'histoire François Bourdon en 2003. Il est par ailleurs professeur d'ESHMC en CPGE voie ECE option Saint-Cyr au Lycée Militaire d'Autun. Spécialiste d'histoire des entreprises et de l'industrie, il a notamment publié La Stratégie des Schneider, du marché à la firme intégrée en 2006, (réed. 2015) et Les Voyages forment l'ingénieur, les houillères du Centre et du Midi de la France, 1851-1873, Paris, Classiques Garnier, en 2015. Avec Marco Bertilorenzi et Anne-Françoise Garçon, il a dirigé les actes du colloque Entre technique et gestion, une histoire des ingénieurs civils des mines, XIX ${ }^{e}-X X^{e}$ siècles, Paris, Presses des Mines, 2016 et achève ceux du colloque Henri 
Fayol, histoire, économie et sciences. Sous le parrainage d'Anne-Françoise Garçon, il prépare actuellement son HDR. 